\title{
Decisiones óptimas de consumo y portafolio:un enfoque de precios de estado de Arrow-Debreu
}

Nora Gavira Durón Instituto Politécnico Nacional, Escuela Superior de Economía aseunam53@yahoo.com.mx

Francisco Venegas

Martínez

Instituto Politécnico Nacional, Escuela Superior de Economía fvenegas1111@yahoo.com.mx

\section{Resumen}

Esta investigación desarrolla, bajo el supuesto de mercados completos, un modelo estocástico que explica el proceso de toma de decisiones de un consumidor-inversionista racional al seleccionar un portafolio en un ambiente de riesgo de mercado sujeto a su restricción presupuestal. El modelo propuesto se desarrolla en el marco de utilidad esperada del tipo Von Neumann-Morgenstern y precios de estado del tipo Arrow-Debreu en un horizonte de planeación infinito. Los principales resultados son: 1) la proporción que el individuo asigna de su riqueza a la tenencia del activo riesgoso es constante y 2) la estrategia óptima de consumo que el agente sigue es que siempre consume la misma proporción de su riqueza.

Palabras clave: comportamiento del consumidor, precios de estado, selección de portafolio, decisiones de inversión.

Clasificación JEL: D11, G11, G13. 


\title{
Optimal Consumption and Portfolio Decisions: an Arrow-Debreu State Princes Approach
}

\begin{abstract}
This research develops, under the assumption of complete markets, a stochastic model that explains the decision making process of a rational consumer-investor selecting a portfolio in a market risk environment subject to his budget constraint. The proposed model is developed in the framework of expected utility of von Neumann-Morgenstern type and state prices of Arrow-Debreu type in an infinite planning horizon. The main results are: 1) the proportion that the individual allocates his wealth to the risky asset holding is constant, and 2) the optimal consumption strategy that the agent follows is consuming always the same proportion of his wealth.
\end{abstract}

Keywords: Consumer economics, contingent pricing, portfolio choice, investment decisions.

JEL Classification: D11, G11, G13.

\section{Introducción}

Aunque, en la literatura especializada, existe una tendencia creciente en el desarro1 lo de métodos estocásticos (matemáticas financieras estocásticas) para optimizar portafolios, es todavía incomprensible ver que el método clásico de media-varianza desarrollado por Markowitz (1952) sigue siendo ampliamente utilizado. La crítica sobre el enfoque media-varianza es bien conocida, por un lado, la varianza no es una medida coherente de riesgo (Artzner, Delbaen, Eber y Heath, 1999) y, por otro, la distribución empírica de los rendimientos, usualmente, presenta una cresta más alta y colas más pesadas que la distribución normal; además del sesgo, los clusters de volatilidad y la heteroscedasticidad condicional (Fama 1965 y Bollerslev 1986). No obstante, la aproximación media-varianza sigue siendo un estándar en la industria financiera, la razón es que el modelo clásico es computacionalmente eficiente; y fácil de programar; mientras que los métodos provenientes de las matemáticas financieras son, todavía para muchos, inaccesibles.

El supuesto de que los rendimientos provienen de una distribución normal es una buena aproximación para divisas de países industrializados. Sin embargo, en las últimas décadas, el desarrollo de los mercados financieros, aunado al impresio- 
nante avance de las tecnologías de información, ha proporcionado muchos y muy diversos instrumentos financieros, incluyendo los productos derivados, las notas estructuradas y los productos con riesgo de crédito, en cuyo caso el uso de la varianza como medida de riesgo ya no es justificable.

Como una alternativa al método clásico se encuentran los resultados de las finanzas estocásticas en el marco de agentes racionales que maximizan utilidad, donde la solución del problema de optimización se presenta a través de un proceso de precios de estado asociados a una medida martingala equivalente (única si los mercados son completos). Y aunque este enfoque es más sofisticado, también hay que reconocer que es mucho más realista (que el enfoque clásico).

La literatura sobre decisiones de consumo y portafolio bajo el criterio de maximización de utilidad tiene su origen en los artículos de Merton (1969) y (1971) en donde el movimiento browniano se utiliza para modelar el riesgo de mercado. Posteriormente, Harrison y Kreps (1979), y Harrison y Pliska (1981) relacionan el concepto de martingala con el problema de decisión de un consumidor-inversionista racional. Así mismo, Cox y Huang (1989) y Venegas-Martínez (2001), (2006a), (2006b) y (2010a) incorporan, en el problema de decisión de maximización de utilidad, saltos de Poisson en la dinámica de los activos riesgosos y Venegas-Martínez (2008a), (2008b) y (2009) generaliza el proceso de Poisson cuando el tamaño del salto es conducido por una distribución de valor extremo de tipo Fréchet. De la misma manera, Basak y Shapiro (2001), y Grossman y Zhou (1996) introducen restricciones sobre la riqueza y su valor en riesgo. Venegas-Martínez y Rodríguez-Nava (2009) estudian la relación entre maximización de utilidad y minimización de varianza de un portafolio. Por otro lado, el proceso de maximización de utilidad con activos con volatilidad estocástica ha sido examinado por Lewis (2000) y Venegas-Martínez (2005). También, Venegas-Martínez (2005) y (2010b) combina saltos de Poisson con volatilidad estocástica. Por último, Ángeles-Castro y Venegas-Martínez (2010) y Venegas-Martínez y Cruz-Ake (2010) recurren a agentes maximizadores de utilidad, en un ambiente de equilibrio general, para valuar productos derivados.

En toda la literatura anterior, el objetivo principal de un consumidor racional que cuenta con una dotación inicial de su riqueza es la asignación de sus recursos al consumo y a los diferentes activos disponibles en los mercados financieros. No obstante, la literatura sobre maximización de utilidad en el marco de utilidad esperada del tipo von Neumann-Morgenstern (1944) y de precios de estado del tipo 
Arrow-Debreu (Arrow, 1953) es escasa. Al respecto, es importante mencionar los trabajos de Karatzas y Shreve (1991), Karatzas, Lehoczky, Sethi, y Shreve (1986), y Karatzas, Lehoczky y Shreve (1986) en el marco de mercados completos y Karatzas, Lehoczky, Shreve y Xu (1991) en caso de mercados incompletos.

Los principales resultados de esta investigación, en el marco de utilidad esperada y precios de estado, son los siguientes: primero, dada la incertidumbre de los posibles estados (de la naturaleza), la cual es independiente de las preferencias y las dotaciones, se muestra que, para hacer frente a la incertidumbre de los mercados financieros) el agente consume siempre una proporción constate de la riqueza disponible (cualquiera que ésta sea). Segundo, una característica sorprendente de la solución es que la proporción que asigna, el agente, de su riqueza a la tenencia del activo riesgoso es invariante en el tiempo (lo mismo para el activo libre de riesgo). Es importante destacar que el primer resultado coincide con el encontrado, en el marco determinista, por Modigliani (1971). Por último, es importante destacar que los resultados obtenidos son también consistentes con el teorema de separación de Fisher (1930).

Este trabajo está organizado de la siguiente forma. En la próxima sección se introducen las alternativas de inversión disponibles para el consumidor-inversionista. En el transcurso de la sección 3, la restricción presupuestal dinámica del agente se transforma en una restricción presupuestal estática, específicamente la restricción equivalente es un valor esperado sobre el horizonte de planeación. En la sección 4 se introduce un sistema de precios de estado del tipo de Arrow-Debreu, es decir, precios de contratos contingentes por unidad de probabilidad $\mathrm{P}$ sobre una unidad de un bien de consumo. En la sección 5 se plantea el problema de decisión del consumidor-inversionista con función de utilidad esperada del tipo von Neumann-Morgenstern y se establecen las condiciones de primer orden (condiciones necesarias) para una solución interior. En la sección 6 se discute el caso de utilidad logarítmica, la cual está asociada a consumidores adversos al riesgo. Por último, en la sección 7 se presentan las conclusiones.

\section{Mercados financieros}

Los mercados financieros son instituciones que permiten a los agentes económicos el intercambio de activos financieros bajo la supervisión de una entidad regulatoria. A continuación se caracterizan los mercados financieros y se detalla la dinámica 
de los activos financieros disponibles en ellos. Asimismo, se supone que todos los activos se cotizan y negocian en términos reales, es decir, en términos de bienes.

En el mercado de dinero, las oportunidades de inversión en instrumentos de deuda (o de renta fija) están representadas por un bono, cupón cero, libre de riesgo de incumplimiento (emitidos por un gobierno) cuyo precio, $B_{t}$, tiene la siguiente dinámica:

$$
\frac{\mathrm{d} B_{t}}{B_{t}}=r \mathrm{~d} t,
$$

donde $r$ es una tasa de interés continuamente capitalizable y constante para todos los plazos, es decir, la estructura temporal de la tasa de interés es plana. Se supone, por simplicidad, que $B_{0}=1$. En cuyo caso, la dinámica del precio del bono es $B_{t}=e^{r t}$.

El riesgo en el mercado de títulos de capital será modelado a través de un espacio de probabilidad equipado con su filtración aumentada $\left(\Omega, \mathcal{F},\left(\mathcal{F}_{t}\right)_{t \in 0, T}, \mathbb{P}\right)$ sobre el cual está definido un movimiento browniano estándar (o un proceso de Wiener), $\mathrm{d} W_{t} \sim \mathcal{N}(0, \mathrm{~d} t)$. En este caso, el proceso $\mathrm{d} W_{t}$ modela los movimientos que se observan todos los días en los rendimientos del título. La filtración $\mathcal{F}_{t}$ representa la información (sobre precios) disponible al tiempo $t$.

En este trabajo se supondrá la existencia de un solo emisor de acciones, la incorporación de dos o más emisores, solamente hace la notación más compleja a cambio de una contribución marginal en el análisis. Así, pues, existe un activo con riesgo (una acción) de precio $S_{t}$ cuya dinámica está representada por la siguiente ecuación diferencial estocástica (difusión):

$$
\frac{\mathrm{d} S_{t}}{S_{t}}=\mu \mathrm{d} t+\sigma \mathrm{d} W_{t},
$$

donde $\mu$ es el rendimiento medio anualizado de la acción y $\sigma$ es la desviación estándar de los rendimientos anualizados alrededor de $\mu$. Los parámetros $\mu$ y $\sigma$ son llamados, respectivamente, de tendencia y volatilidad. 


\section{Restricción presupuestal del agente}

Se supone que el agente tiene una dotación inicial de riqueza, en términos de bienes, $a_{0}$. Asimismo, se supone que la riqueza real del individuo está dada por el portafolio

donde

$$
\Pi_{t} \equiv A_{t}=B_{t}+S_{t}
$$

$$
\Pi_{0}=A_{0}=B_{0}+S_{0}
$$

es dado. Los cambios marginales en el valor del portafolio se deben exclusivamente a cambios marginales en los precios de los activos, de tal manera que

$$
\mathrm{d} \Pi_{t}=\mathrm{d} B_{t}+\mathrm{d} S_{t}=A_{t}\left(1-\theta_{t}\right) \frac{\mathrm{d} B_{t}}{B_{t}}+A_{t} \theta_{t} \frac{\mathrm{d} S_{t}}{S_{t}}
$$

donde $\theta_{t}=S_{t} / A_{t} \quad$ y $1-\theta_{t}=B_{t} / A_{t} \quad$ son, respectivamente, la proporción de la riqueza real que se asigna a la tenencia del activo riesgoso y la proporción, complementaria, que se destina al compra de bonos. Para calcular el cambio marginal de la riqueza, $\mathrm{d} A_{t}$, se requiere restar el consumo por unidad del tiempo del valor del portafolio, es decir,

$$
\mathrm{d} A_{t}=\mathrm{d} \Pi_{t}-\mathrm{d} c_{t},
$$

Después de sustituir las ecuaciones (1) y (2) en la expresión (6) y de escribir $\mathrm{d} c_{t}=c_{t} \mathrm{~d} t$, se tiene que (6) se transforma en:

$$
\mathrm{d} A_{t}=A_{t}\left[r+\theta_{t}(\mu+\delta-r)-\frac{c_{t}}{A_{t}}\right] \mathrm{d} t+A_{t} \theta_{t} \sigma \mathrm{d} W_{t}
$$

Es conveniente definir ahora el premio al riesgo de mercado:

$$
\lambda \equiv \frac{\mu-r}{\sigma}
$$


Como puede observarse, para que este premio sea lo más grande posible se requiere no sólo que el rendimiento medio, $\mu$, de un mundo con riesgo sea mayor que el rendimiento, $r$, de un mundo seguro, sino también que la volatilidad, $\sigma$, sea lo más pequeña posible, obligando con ello a que los posibles rendimientos del activo riesgoso se mantengan cercanos a $\mu$. La cantidad $\lambda$ también es conocida como la razón de Sharpe (1964) y es la herramienta principal en la selección de portafolios. Por ejemplo, si esta razón se calcula para varios activos riesgosos, basta seleccionar a aquellos para los cuales dicha razón sea mayor (el criterio de selección es que la varianza del portafolio sea mínima). La grafica 1 muestra el comportamiento del premio al riesgo de mercado en función de $\mu$ y $\sigma$ en donde se ha tomado $r=6 \%$.

\section{Gráfica 1}

Comportamiento del $\lambda$ en función de $\mu$ y $\sigma$.

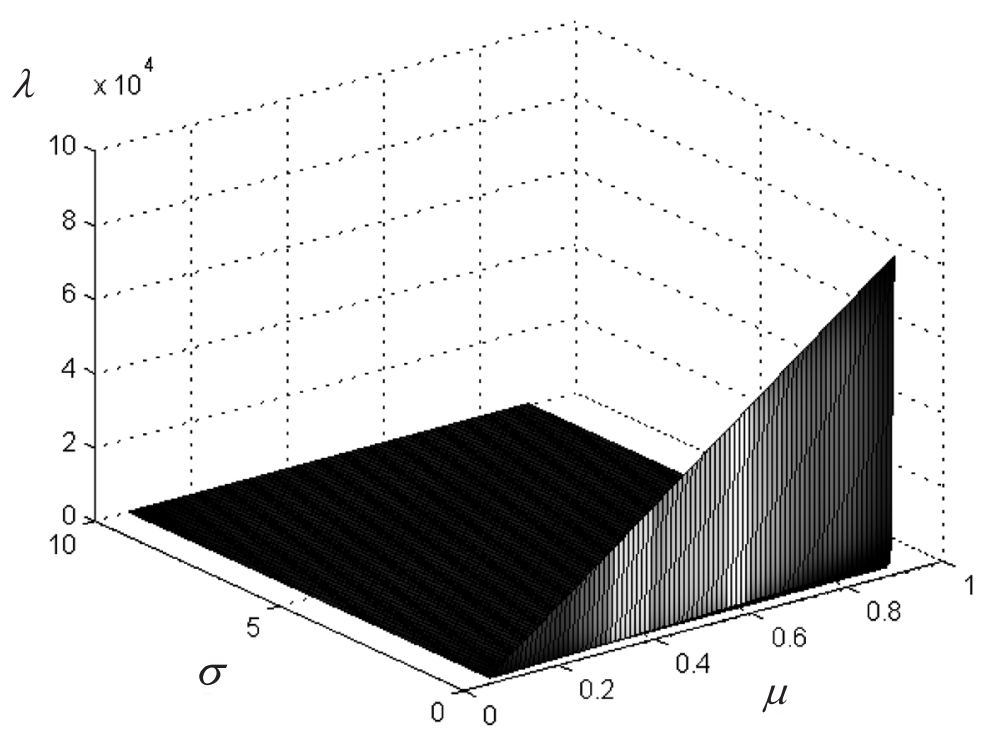

En lo que sigue se examina la relación entre riesgo y rendimiento expresado en (8). A continuación se obtiene la proporción (óptima) que el agente tiene que asignar a la tenencia del activo riesgoso. En principio, el valor óptimo de $\theta_{t}$ debería depender sólo de las características del mercado, específicamente, de la varianza (vista 
como el riesgo) y del rendimiento del activo riesgoso, y no las preferencias por el consumo expresadas en la función de utilidad y en la tasa subjetiva de descuento del consumo del agente. Por lo tanto, la información en la dinámica de los activos, expresada en la restricción presupuestal, debería ser suficiente para determinar las proporciones óptimas de la riqueza que se deben asignar a los activos en el portafolio. En lo que sigue se obtiene el valor de $\theta_{t}$ que maximiza el rendimiento (logarítmico) medio esperado del portafolio, el cual está dado por:

$$
\mathrm{E}^{\mathrm{P}}\left[\mathrm{d} \ln \left(\frac{\Pi_{t}}{\Pi_{0}}\right) \mid \mathcal{F}_{t}\right]=\mathrm{E}^{\mathrm{P}}\left[\mathrm{d}\left(\ln \left(\Pi_{t}\right)-\ln \left(A_{0}\right)\right] \mid \mathcal{F}_{t}\right]=\mathrm{E}^{\mathrm{P}}\left[\mathrm{d} \ln \left(A_{t}\right) \mid \mathcal{F}_{t}\right] .
$$

Si se aplica el lema de Itô a $\ln \left(\Pi_{t}\right)$, tomando como proceso subyacente en cambio marginal en el valor del portafolio, dado en (5), se obtiene que

$$
\mathrm{d} \ln \left(\Pi_{t}\right)=\left(r+\theta_{t} \lambda \sigma-\frac{1}{2} \theta_{t}^{2} \sigma^{2}\right) \mathrm{d} t+\theta_{t} \sigma \mathrm{d} W_{t}
$$

y en consecuencia el rendimiento esperado del portafolio satisface

$$
\mathrm{E}\left[\mathrm{d} \ln \left(\Pi_{t}\right) \mid \mathcal{F}_{t}\right]=\left(r+\gamma+\theta_{t} \lambda \sigma-\frac{1}{2} \theta_{t}^{2} \sigma^{2}\right) \mathrm{d} t
$$

Si se elige $\theta_{t}$ de tal manera que se maximice el rendimiento del portafolio por unidad de tiempo, dado por

$$
G\left(\theta_{t}\right) \equiv \mathrm{E}\left[\mathrm{d} \ln \left(\Pi_{t}\right) \frac{1}{\mathrm{~d} t} \mid \mathcal{F}_{t}\right]
$$

la condición $G^{\prime}\left(\theta_{t}\right)=0$ conduce al máximo

$$
\theta_{t} \equiv \lambda / \sigma
$$


ya que $G^{\prime \prime}\left(\theta_{t}\right)=-\sigma^{2}<0$. La cantidad (9), además de maximizar el rendimiento del portafolio, mantiene la volatilidad (desviación estándar del rendimiento de la riqueza) del rendimiento del portafolio constante. Así, la determinación de $\theta_{t}$ toma sólo en cuenta la dinámica de los activos en el portafolio, omitiendo el consumo y las preferencias por el mismo. De tal manera que se separan las decisiones de portafolio de las de consumo, tal como lo sugiere el teorema de separación de Fisher (1930); véase, por ejemplo, Venegas-Martínez (2008a, p. 985).

La ecuación diferencial estocástica en (7) que conduce la dinámica de la riqueza real no incorpora explícitamente el horizonte de planeación, es decir, no incorpora explícitamente el intervalo $[0, \infty)$. A continuación se reescribe (7) de tal manera que se pueda incluir un horizonte de planeación infinito. La ecuación resultante se expresa en términos de integrales definidas en $[0, \infty)$, y recibirá el nombre de restricción presupuestal estática. Considere el proceso (el precio estocástico del bien)

$$
Z_{t}=\exp \left\{-\frac{1}{2} \lambda^{2} t-\lambda W_{t}\right\} .
$$

Es fácil verificar que este proceso satisface la siguiente ecuación diferencial estocástica

$$
\mathrm{d} Z_{t}=-\lambda Z_{t} \mathrm{~d} W_{t}
$$

Observe también que, a partir de (7), se puede escribir

$$
\mathrm{d} A_{t}=\left(A_{t} r+A_{t} \theta_{t} \sigma \lambda-c_{t}\right) \mathrm{d} t+A_{t} \theta_{t} \sigma \mathrm{d} W_{t} .
$$

Sea

$$
H_{t}=e^{-r t} Z_{t} \text {. }
$$

Si se aplica el lema de Ito Itô a $A_{t} H_{t}$, se tiene que

$$
\mathrm{d}\left(A_{t} H_{t}\right)=-H_{t} c_{t} \mathrm{~d} t+A_{t} H_{t}\left(\theta_{t} \sigma-\lambda\right) \mathrm{d} W_{t}
$$


es decir,

$$
\lim _{s \rightarrow \infty} A_{s} H_{s}-A_{t} H_{t}=-\int_{t}^{\infty} H_{s} c_{s} \mathrm{~d} s+\int_{t}^{\infty} H_{s} A_{s}\left(\theta_{s} \sigma-\lambda\right) \mathrm{d} W_{s} .
$$

Si se elige $\theta_{t}$ de tal manera que se anule la última integral en (15) a fin de eliminar el factor de riesgo $\mathrm{d} W_{s}$, se tiene que

$$
\theta_{t}=\lambda / \sigma
$$

Si se supone además la condición de transversalidad

$$
\lim _{s \rightarrow \infty} A_{s} H_{s}=0
$$

después de tomar esperanzas, se tiene que

$$
\mathrm{E}^{\mathrm{P}}\left[\int_{t}^{\infty} H_{s} c_{s} \mathrm{~d} s \mid \mathcal{F}_{t}\right]=H_{t} A_{t}
$$

Esto separa las decisiones de inversión de las de consumo, lo cual está de acuerdo con teorema de separación de Fisher (1930). Por supuesto, una vez que se tengan las decisiones óptimas la condición de transversalidad se tiene que verificar.

\section{Precios de estado de Arrow-Debreu}

A continuación se introducen los precios de Arrow-Debreu de contratos contingentes por unidad de probabilidad $\mathrm{P}$ sobre una unidad de un bien de consumo. Estos precios de estado se obtienen en condiciones de equilibrio de los mercados financieros y, en consecuencia, no existen posibilidades arbitraje (ganancias libres de riesgo). Ahora bien, si la restricción presupuestal, dada en (17), se reescribe como

$$
\mathrm{E}^{\mathrm{P}}\left[\int_{0}^{\infty} Z_{t} c_{t} e^{-r t} \mathrm{~d} t \mid \mathcal{F}_{0}\right]=A_{0}
$$


ó

$$
\mathrm{E}^{\mathrm{P}}\left[\int_{0}^{\infty}\left(Z_{t} c_{t}-A_{0} r\right) e^{-r t} \mathrm{~d} t \mid \mathcal{F}_{0}\right]=0
$$

donde $Z_{t}$ se toma como en (10), entonces el proceso $Z_{t}$ puede ser interpretado como el precio de Arrow-Debreu por unidad de probabilidad $P$ de una unidad de un bien de consumo en el tiempo $t$. En otras palabras, $Z_{t}$ es el precio de un contrato contingente en el que si cierto estado de la naturaleza ocurre entonces el contrato paga una unidad del bien en una fecha preestablecida. La función de densidad asociada a los posibles estados de la naturaleza es la densidad de la variable aleatoria $Z_{t}$; su función de densidad se determinará posteriormente. Asimismo, se puede mostrar, de manera muy sencilla, que $Z_{t}$ es una martingala con respecto de $\mathrm{P}$ (véase el Apéndice). Es decir, $Z_{t}$ satisface $\mathrm{E}^{\mathrm{P}}\left[Z_{s} \mid F_{t}\right]=Z_{t}, s \geq t$. En otras palabras, el mejor pronóstico para $Z_{s}$, dado que se conoce $Z_{t}$, es $Z_{t}$. Esto es, el mejor pronóstico para el precio de mañana, es el precio de hoy (hipótesis de mercados eficientes, véase Fama 1970). El precio $Z_{t}$ es la única (medida) martingala neutral al riesgo, tal que $\mathrm{d} \tilde{\mathrm{P}}=Z_{t} \mathrm{dP}$, donde $\mathrm{d} \tilde{W}_{t}=\lambda \mathrm{d} t+\mathrm{d} W_{t} \sim \mathcal{N}(0, \mathrm{~d} t)$ es un movimiento browniano definido en un espacio de probabilidad $\left(\Omega, \mathcal{F},\left(\mathcal{F}_{t}\right)_{t \in 0, T}, \tilde{\mathbb{P}}\right) . \quad Z_{t}$ es única porque los mercados son completos, es decir, existe el mismo número de factores de riesgo (un movimiento browniano) que de activos riesgosos que se cotizan en el mercado (una acción) que satisface (19) y es neutral al riesgo porque la tendencia de la restricción $\mathrm{d} A_{t}=\left(A_{t} r-c_{t}\right) \mathrm{d} t+\sigma A_{t} \theta_{t} \mathrm{~d} \tilde{W}_{t}$, sólo depende de la tasa de interés libre de riesgo de incumplimiento y no del rendimiento $\mu$. También se puede ver, fácilmente, que el proceso $H_{t}=Z_{t} e^{-r t}=Z_{t} / B_{t}$ satisface la ecuación diferencial estocástica:

$$
\mathrm{d} H_{t}=-H_{t} r \mathrm{~d} t+\lambda \mathrm{d} W_{t}
$$

En este caso, una simple aplicación del lema Itô a $\ln \left(H_{t}\right)$ conduce a

$$
H_{s}=H_{t} \exp \left\{-\left(r+\frac{1}{2} \lambda^{2}\right)(s-t)-\lambda\left(W_{s}-W_{t}\right)\right\}, \quad s \geq t
$$


La función de densidad $H_{s}$, dado $H_{t}$, es log-normal y está dada por

$$
f_{H_{s} \mid H_{t}}\left(x \mid H_{t}\right)=\frac{1}{\sqrt{2 \pi(s-t)} \lambda x} \exp \left\{-\frac{1}{2}\left(\frac{\ln \left(\frac{x}{H_{t}}\right)+\left(r+\frac{1}{2} \lambda^{2}\right)(s-t)}{\lambda \sqrt{s-t}}\right)^{2}\right\}, x>0 .
$$

Asimismo, dado que $Z_{t}=H_{t} e^{r t}$ es el precio de un contrato contingente que hace un pago en términos de bienes en una fecha preestablecida. Por último, la dinámica del precio de activo riesgoso, dado en (2), en términos de la densidad de precios de estado es equivalentes a

$$
H_{t} S_{t}=\mathrm{E}^{\mathrm{P}}\left[H_{u} S_{u} \mid \mathcal{F}_{t}\right], \quad u \geq t,
$$

\section{Problema de decisión del consumidor}

Considere un consumidor-inversionista con preferencias y dotaciones exógenas que toma decisiones en un horizonte infinito, $[0, \infty)$. Se supone que el individuo maximiza su satisfacción por un bien genérico de consumo de carácter perecedero. Asimismo, se supone que el individuo es adverso al riesgo y tomador de precios.

El agente está dotado con una riqueza inicial, $A_{0}$, y elige, en cada instante, de su horizonte de planeación $[0, \infty)$, la proporción de su riqueza que asignará a la tenencia de un bono libre de riesgo de incumplimiento y la proporción que destinará a un activo riesgoso. Se puede pensar en un individuo cuyo horizonte de vida es el intervalo $[0, \infty)$ o como un padre que está interesado en maximizar la utilidad de sus hijos, nietos y demás descendientes, entre otras posibilidades.

En la economía hay disponible un solo bien genérico de consumo (el numerario). El consumidor obtiene satisfacción por el bien de consumo, $c_{t}$. La utilidad esperada del tipo von Neumann-Morgenstern, $V_{0}$, al tiempo $t=0$, del individuo, el cual se supone adverso al riesgo y competitivo (tomador de precios), está dada por:

$$
V_{0} \equiv \mathrm{E}^{\mathrm{P}}\left[\int_{0}^{\infty} u\left(c_{t}\right) e^{-\rho t} \mathrm{~d} t \mid \mathcal{F}_{0}\right]
$$


donde $\rho$ es la tasa subjetiva de descuento (o tasa de descuento intertemporal). Esta tasa es un indicador de que tan ansioso está el individuo por el consumo presente. En otras palabras entre mayor sea $\rho$, más ansioso estará el agente por consumir en el presente que en el futuro). Como siempre, se supone que la función de utilidad $u\left(c_{t}\right)$ es dos veces continuamente diferenciable con

$$
u^{\prime}\left(c_{t}\right)>0
$$

y

$$
u^{\prime \prime}\left(c_{t}\right)<0 \text {. }
$$

Observe que la forma funcional de la utilidad no cambia en todo el horizonte de planeación, esto es para suavizar el consumo en el tiempo.

Suponga que el agente está dotado, al tiempo $t=0$, con una riqueza inicial de $A_{0}$. A continuación se plantea el problema de un consumidor que maximiza utilidad por el bien de consumo donde en lugar de utilizar la restricción (7) se utiliza su equivalente en función de la densidad de precios de estado, $H_{t}$, dada en (17):

$$
\underset{c_{t}}{\operatorname{Maximizar}} \quad \mathrm{E}^{\mathrm{P}}\left[\int_{0}^{\infty} u c_{t} e^{-\rho t} \mathrm{~d} t \mid \mathcal{F}_{0}\right]
$$

sujeto a: $\quad \mathrm{E}^{\mathrm{P}}\left[\int_{0}^{\infty}\left(Z_{t} c_{t}-A_{0} r\right) e^{-r t} \mathrm{~d} t \mid \mathcal{F}_{0}\right]=0, \quad A_{0}>0 \quad$ dado.

Es importante destacar que la solución del problema de maximización de utilidad no proveerá una trayectoria óptima de consumo, sino que dará una regla óptima sobre la proporción de la riqueza que se debe consumir en cada instante en función de los parámetros de preferencias, la dotación inicial y los parámetros que caracterizan las dinámicas de los activos. En este caso, el Lagrangeano del problema (26) es

$$
L \equiv \mathrm{E}^{\mathrm{P}}\left[\int_{0}^{\infty}\left[u\left(c_{t}\right) e^{-\rho t}-\eta H_{t} c_{t}+A_{0} r e^{-r t}\right] \mathrm{d} t \mid \mathcal{F}_{0}\right]
$$


donde $\eta$ es el multiplicador (constante) de Lagrange asociado a la restricción. Una condición necesaria para que $\partial L / \partial c_{t}=0$, es que el Lagrangeano asociado, $\mathcal{L}$ a la parte del integrando que depende de la variable de decisión $c_{t}$ :

satisfaga

$$
\mathcal{L} \equiv u\left(c_{t}\right) e^{-\rho t}-\eta H_{t} c_{t}
$$

$$
0=\frac{\partial \mathcal{L}}{\partial c_{t}}=u^{\prime}\left(c_{t}\right) e^{-\rho t}-\eta H_{t},
$$

es decir, la utilidad marginal es proporcional al precio de estado, $H_{t}$. En consecuencia, el consumo óptimo satisface

$$
c_{t}=I\left(\eta H_{t} e^{\rho t}\right) .
$$

donde $I$ es la función inversa de la utilidad marginal. Así, el consumo depende de las preferencias del agente (expresadas a través de la función de utilidad), de los precios de estado y del multiplicador (el cual se obtendrá endógenamente).

\section{Utilidad logarítmica}

Con el propósito de ilustrar los resultados de la sección anterior se supondrá ahora una forma funcional específica del índice de satisfacción. En el análisis subsiguiente la función de utilidad estará dada por:

$$
u\left(c_{t}\right)=\ln \left(c_{t}\right)
$$

En este caso, la condición necesaria de óptimo, expresada en (27), conduce a

$$
c_{t}=\frac{e^{-\rho t}}{\eta H_{t}}
$$

Observe ahora, de acuerdo con (17), que

$$
A_{t}=H_{t}^{-1} \mathrm{E}^{\mathrm{P}}\left[\int_{t}^{\infty} H_{u} c_{u} \mathrm{~d} u \mid \mathcal{F}_{t}\right]
$$


En este caso, al sustituir (29) en (30), se obtiene que

$$
A_{t}=H_{t}^{-1} \mathrm{E}^{\mathrm{P}}\left[\int_{t}^{\infty} H_{u} c_{u} \mathrm{~d} u \mid \mathcal{F}_{t}\right]=H_{t}^{-1} \mathrm{E}^{\mathrm{P}}\left[\int_{t}^{\infty} \eta^{-1} e^{-\rho u} \mathrm{~d} u \mid \mathcal{F}_{t}\right]=\frac{\left(\eta H_{t}\right)^{-1} e^{-\rho t}}{\rho}=\frac{c_{t}}{\rho} .
$$

Es decir, el consumo es proporcional a la riqueza y la constante de proporcionalidad es $\rho$ Observe, por último que a partir de (29) y (31) se verifica la condición de transversalidad,

$$
\lim _{s \rightarrow \infty} A_{s} H_{s}=A_{0} \lim _{s \rightarrow \infty} e^{-\rho s}=0,
$$

Falta determinar $\eta$ (el precio sombra del consumo) a fin de que (29) quede expresada solamente en términos de variables exógenas. Observe primero, que a partir de (17), se obtiene

$$
A_{0}=\mathrm{E}^{\tilde{\mathrm{P}}}\left[\int_{0}^{\infty} H_{u} c_{u} \mathrm{~d} u \mid \mathcal{F}_{0}\right]=\mathrm{E}^{\tilde{\mathrm{P}}}\left[\int_{0}^{\infty} \eta^{-1} e^{-\rho u} \mathrm{~d} u \mid \mathcal{F}_{0}\right]=(\eta \rho)^{-1}:
$$

lo cual implica que

$$
\eta=\frac{1}{\rho A_{0}}
$$

Así, el multiplicador $\eta$ depende de la dotación del agente $A_{0}$ y del parámetro de preferencia $\rho$ Si se utiliza (29), se obtiene

ó

$$
c_{t}=e^{-\rho t}\left(\eta H_{t}\right)^{-1}=\rho A_{0} e^{(r-\rho) t} \exp \left\{\frac{1}{2} \lambda^{2} t+\lambda W_{t}\right\}
$$

$$
c_{t}=c_{0} \exp \left\{\left(\mu_{c}-\frac{1}{2} \sigma_{c}^{2}\right) t+\sigma_{c} W_{t}\right\} \text {. }
$$

donde

$$
c_{0}=\rho A_{0}, \quad \mu_{c}=r-\rho+\lambda^{2} \quad \text { y } \quad \sigma_{c}=\lambda,
$$


aquí $\mu_{c}$ es el parámetro de tendencia física del consumo y $\sigma_{c}$ es la volatilidad instantánea del mismo. Observe también que una expresión equivalente a (35), en términos de una ecuación diferencial estocástica (de una difusión) es

$$
\frac{\mathrm{d} c_{t}}{c_{t}}=\mu_{c} \mathrm{~d} t+\sigma_{c} \mathrm{~d} W_{t} .
$$

Como puede observarse, a partir de (34), cuando el agente es expuesto al riesgo de mercado, dicho riesgo afecta de manera sensible su comportamiento y expectativas. En el marco determinista, el individuo puede prescribir, con toda seguridad, cual será su trayectoria óptima de consumo. En el caso estocástico, infortunadamente, la trayectoria de consumo ya no puede ser determinada porque el consumo se convierte en variable aleatoria, situación que está más acorde con la realidad. De acuerdo con (35), el valor esperado del consumo, al tiempo $t$,

$$
\mathrm{E}^{\mathrm{P}}\left[c_{t} \mid \mathcal{F}_{0}\right]=c_{0} \exp \left\{\left(r-\rho+\lambda^{2}\right) t\right\}
$$

y su varianza está dada por

$$
\operatorname{Var}^{\mathrm{P}}\left[c_{t} \mid \mathcal{F}_{0}\right]=c_{0}^{2} \exp \left\{2\left(r-\rho+\lambda^{2}\right) t\right\}\left[\exp \left\{\left(r-\rho+\lambda^{2}\right)^{2} t\right\}-1\right] .
$$

La función de densidad del consumo está dada por

$$
f_{c_{t} \mid c_{0}}\left(x \mid c_{0}\right)=\frac{1}{\sqrt{2 \pi t} \sigma_{c} x} \exp \left\{-\frac{1}{2}\left(\frac{\ln \left(\frac{x}{c_{0}}\right)-\left(\mu_{c}-\frac{1}{2} \sigma_{c}^{2}\right) t}{\sigma_{c} \sqrt{t}}\right)^{2}\right\} .
$$

En virtud de que el consumo es una variable aleatoria, sólo puede determinarse la probabilidad de que el consumo se encuentre en un intervalo dado $[\underline{c}, \bar{c}]$. De esta forma, al utilizar (40), se tiene que

$$
\mathbb{P}\left\{\underline{c} \leq c_{t} \leq \bar{c} \mid \mathcal{F}_{0}\right\}=\operatorname{E}^{\mathrm{P}}\left[1_{[\underline{c}, \bar{c}]} \mid \mathcal{F}_{0}\right]=\int_{\underline{c}}^{\bar{c}} f_{c_{t} \mid c_{0}}\left(x \mid c_{0}\right) \mathrm{d} x .
$$


Se puede ver que

$$
\mathrm{P}\left\{c_{t} \geq \bar{c} \mid \mathcal{F}_{0}\right\}=\int_{\bar{c}}^{\infty} f_{c_{t} \mid c_{0}}\left(x \mid c_{0}\right) \mathrm{d} x=\Phi \bar{d}
$$

donde

$$
\bar{d}=\frac{\ln \left(\frac{c_{0}}{\bar{c}}\right)+\left(r-\rho+\frac{1}{2} \lambda^{2}\right) t}{\sigma_{c} \sqrt{t}} .
$$

De esta manera,

$$
\mathrm{P}\left\{\underline{c} \leq c_{t} \leq \bar{c} \mid \mathcal{F}_{0}\right\}=\Phi(\underline{d})-\Phi(\bar{d})
$$

donde, de manera similar,

$$
\underline{d}=\frac{\ln \left(\frac{c_{0}}{\underline{c}}\right)+\left(r-\rho+\frac{1}{2} \lambda^{2}\right) t}{\sigma_{c} \sqrt{T}} .
$$

Para determinar, de manera alternativa, la proporción óptima de la riqueza que se asignará a la tenencia del activo riesgoso, note que a partir de (29) y (31)

$$
A_{t} H_{t}=A_{0} e^{-\rho t}
$$

lo cual conduce a

$$
\mathrm{d} \ln \left(A_{t}\right)=-\mathrm{d} \ln \left(H_{t}\right)-\rho \mathrm{d} t .
$$

Es decir, la tasa de crecimiento de la riqueza en el equilibrio, es igual a la diferencia entre la tasa de crecimiento de los precios (de estado) y la tasas subjetiva de descuento y de proporcionalidad. Note que a partir del lema de Itô, aplicado tanto en (20) como en (7), se sigue que 


$$
-\mathrm{d} \ln \left(H_{t}\right)=\left(r+\frac{1}{2} \lambda^{2}\right) \mathrm{d} t+\lambda \mathrm{d} W_{t}
$$

$$
\mathrm{d} \ln \left(A_{t}\right)=\left(r+\theta_{t} \sigma \lambda-\frac{1}{2} \theta_{t}^{2} \sigma^{2}-\rho\right) \mathrm{d} t+\theta_{t} \sigma \mathrm{d} W_{t}
$$

En virtud de (47), las componentes estocásticas de (48) y (49) tienen que ser iguales, así

$$
\theta_{t} \sigma \mathrm{d} W_{t}=\lambda \mathrm{d} W_{t}
$$

lo cual implica que $\theta_{t}=\lambda / \sigma$. Por otro lado, si se igualan las componentes deterministas de (48) y (49) y se sustituye $\theta_{t}=\lambda / \sigma$. se obtiene una identidad.

\section{Conclusiones}

Muchos de los portafolios que se operan, actualmente, consideran bonos libres de riesgo de incumplimiento (emitidos por un gobierno) y títulos de capital (emitidos por corporativos), pero muy poco se ha hecho en cuanto al estudio de precios de estado. El presente trabajo ha desarrollado un modelo estocástico que describe el proceso de toma de decisiones de un consumidor-inversionista racional cuando integra un portafolio en el marco de utilidad esperada del tipo von Neumann-Morgenstern y de precios de estado del tipo Arrow-Debreu.

Asimismo, el modelo muestra que la mejor forma para enfrentar la incertidumbre en la selección de un portafolio es que la proporción de la riqueza que se asigna a la tenencia del activo riesgoso se mantenga constante en el tiempo. Aunque el consumo se va modificando en el transcurso del tiempo, no así la proporción de la riqueza que se asigna a la tenencia del activo riesgoso.

En el modelo estocástico propuesto, la trayectoria de consumo no puede ser determinada porque el consumo se convierte en variable aleatoria, situación que es más acorde con la realidad. Así, pues, la consideración del riesgo condujo a cambios cualitativos y cuantitativos importantes en las decisiones de consumo y portafolio. Por último, aunque en este trabajo se examinó una forma funcional particular del 
índice de utilidad, el análisis podría extenderse hacia otras especificaciones alternativas. Por supuesto, la extensión hacia una tasa de interés estocástica ya está en la agenda de investigación.

\section{Apéndice}

Si $s \geq t$, entonces

$$
\begin{aligned}
\mathrm{E}^{\mathrm{P}}\left[\frac{Z_{s}}{Z_{t}} \mid \mathcal{F}_{t}\right] & =\mathrm{E}^{\mathrm{P}}\left[\frac{H_{s}}{H_{t}} \mid \mathcal{F}_{t}\right] e^{r(s-t)} \\
& =\mathrm{E}^{\mathrm{P}}\left[\exp \left\{-\left(r+\gamma+\frac{1}{2} \lambda^{2}\right)(s-t)-\lambda\left(W_{s}-W_{t}\right)\right\} \mid \mathcal{F}_{t}\right] e^{r(s-t} \\
& =\exp \left\{-\left(r+\gamma+\frac{1}{2} \lambda^{2}\right)(s-t)+\frac{1}{2} \lambda^{2}(s-t)\right\} e^{r(s-t)}=1 .
\end{aligned}
$$

De esta manera, $\mathrm{E}^{\mathrm{P}}\left[Z_{s} \mid F_{t}\right]=Z_{t}$.

\section{Bibliografía}

Ángeles-Castro, G. y F. Venegas-Martínez (2010). Valuación de opciones sobre índices bursátiles y determinación de la estructura de plazos de la tasa de interés en un modelo de equilibrio general. Investigación Económica, Vol. 69, No. 271, pp. 43-80.

Arrow, K. J. (1953). The Role of Securities in the Optimal Allocation of RiskBearing, Review of Financial Studies, Vol. 31, No. 2, pp. 91-96.

Artzner, P., F. Delbaen, J. M. Eber, and D. Heath (1999). Coherent Measures of Risk. Mathematical Finance, Vol. 9, No. 3, pp. 203-228. 
Basak, S. and A. Shapiro, (2001), Value-at-Risk-Based Risk Management: Optimal Policies and Asset Prices, The Review of Financial Studies, Vol. 14. No. 2, pp. 371-405.

Bollerslev, T. (1986). Generalized Autoregressive Conditional Heteroskedasticity. Journal of Econometrics, Vol 31, No. 3, pp. 307-327.

Cox, J. C. and C.F. Huang (1989). Optimal consumption and portfolio policies when asset prices follow a diffusion process. Journal of Economic Theory, Vol. 49, No. 1, pp. 33-83.

Fame, E. (1965). The Behavior of Stock Market Prices. Journal of Business, Vol. 38, No. 1. pp. 34-105.

Fame, E. (1970).'Efficient Capital Markets: A Review of Theory and Empirical Work. The Journal of Finance, Vol. 25, No. 2, pp. 383-417.

Fisher, I. (1930). The Theory of Interest. New York: Macmillan.

Grossman, S. J. and Z. Zhou (1996). Equilibrium Analysis of Portfolio Insurance. Journal of Finance, Vol. 51, No. 4, pp. 1379-1403.

Harrison, J. M. and D. M. Kreps (1979), Martingales and Arbitrage in Multiperiod Security Markets, Journal of Economic Theory, Vol. 20, No. 3, pp. 381-408.

Harrison J. M. and S. R. Pliska (1981), Martingales and Stochastic Integrals in The Theory of Continuous Trading, Stochastic Processes and their Applications, Vol. 11, No. 3, pp. 215-260.

Karatzas, I. and S. E. Shreve (1991). Brownian Motion and Stochastic Calculus. Second Edition, Springer-Verlag, New York.

Karatzas, I., J. P. Lehoczky, S. P. Sethi, and S. E. Shreve (1986), Explicit Solution of a General Consumption Problem, Mathematics of Operations Research, Vol. 11, No. 2, pp. 261-294. 
Karatzas, I., J. P. Lehoczky, and S. E. Shreve (1986). Optimal Portfolio And Consumption Decisions For A "Small Investor" On A Finite Horizon, SIAM J. Control and Optimization, Vol. 25, No. 6, pp. 1557-1586.

Karatzas, I., Lehoczky, J. P., Shreve, S. E. and Xu, G. L. (1991). Martingale and Duality Methods for Utility Maximization in an Incomplete Market. SIAM J. Control and Optimizatio, Vol. 29, No. 3. pp. 702-730.

Lewis, A. L. (2000). Option Valuation under Stochastic Volatility: with Mathematica Code, Finance Press. U. K.

Markowitz, H. M. (1952). Portfolio Selection, Journal of Finance, Vol. 7, No.1, pp.77-91.

Merton, R. C. (1969). Lifetime Portfolio Selection under Uncertainty: the Continuous-time Case, Review of Economics and Statistics, Vol. 51, No. 3, pp. 247-257.

Merton, R. C. (1971). Optimum Consumption and Portfolio Rules in a Continuous-Time Model, Journal of Economic Theory, Vol. 3, No. 4, pp. 373-413.

Modigliani, F. (1971). Monetary Policy and Consumption, in Consumer Spending and Monetary Policy: the Linkages. Federal Reserve Bank of Boston, Boston.

Sharpe, W. F. (1964). Capital Asset Prices: A Theory of Market Equilibrium under Conditions of Risk. Journal of Finance, Vol. 19, No. 3, pp. 425-442.

Venegas-Martínez, F. (2001). Temporary Stabilization: A Stochastic Analysis. Journal of Economic Dynamics and Control, Vol. 25, No. 9, pp. 1429-1449.

(2005). Bayesian Inference, Prior Information on Volatility, and Option Pricing: A Maximum Entropy Approach. International Journal of Theoretical and Applied Finance, Vol.8, No. 1, pp. 1-12.

(2006a). Stochastic Temporary Stabilization: Undiversifiable Devaluation and Income Risks. Economic Modelling, Vol. 23, No. 1, pp. 157-173. 
(2006b). Fiscal Policy in a Stochastic Temporary Stabilization Model: undiversifiable Devaluation Risk. Journal of World Economic Review, Vol. 1, No. 1, pp. 13-38.

(2008a). Riesgos financieros y económicos (productos derivados y decisiones económicas bajo incertidumbre), 2da. edición, Cengage Editors (anteriormente International Thomson Editors), México.

(2008b). Real Options on Consumption in a Small Open Monetary Economy. Journal of World Economic Review, Vol. 3, No. 2, pp. 105-115.

(2009). Temporary Stabilization in Developing Countries and Real Options on Consumption. International Journal of Economic Research, Vol. 6, No. 2, 237-257.

(2010a). Fiscal Policy in a Stochastic Model of Endogenous Growth: the Mexican Case. Indian Development Review: An International Journal of Development Economics, Vol. 8, No.1-2, pp. 139-157.

(2010b). Planes no creíbles de estabilización de precios, riesgo cambiario y opciones reales para posponer consumo: un análisis con volatilidad estocástica. El Trimestre Económico, Vol. 77, por aparecer.

y A. Rodríguez-Nava (2009). Consistencia entre minimización de varianza y maximización de utilidad en la valuación de derivados. Revista Contaduría y Administración, No. 229, pp. 9-30.

y S. Cruz-Ake (2010). Productos derivados sobre bienes de consumo. EconoQuantum, Revista de Economía y Negocios, Vol. 6, No. 2, pp. 25-54.

Von Neumann, J. and O. Morgenstern (1944), Theory of Games and Economic Behavior. Princeton University Press, Princeton, N. J. 